\title{
Skull base surgery for tumors in children: long-term clinical and functional outcome
}

\author{
Clinical article
}

\author{
Caroline Hayhurst, F.R.C.S., ${ }^{1}$ Dawn Williams, M.Sc., ${ }^{1}$ Jawad Yousaf, M.R.C.S., ${ }^{1}$ \\ David Richardson, F.R.C.S., ${ }^{2}$ Barry Pizer, F.R.C.P.C.H., Ph.D., ${ }^{3}$ \\ and Conor Mallucci, F.R.C.S. ${ }^{1}$
}

${ }^{1}$ Department of Neurosurgery, ${ }^{2}$ Craniofacial Unit, and ${ }^{3}$ Department of Pediatric Oncology, Alder Hey Children's NHS Foundation Trust, Liverpool, United Kingdom

\begin{abstract}
Object. Skull base tumors in children are rare but require complex approaches with potential morbidity to the developing craniofacial skeleton, in addition to tumor-related morbidity. Reports of long-term clinical and functional outcome following skull base approaches in children are scarce. The authors report long-term outcome in children with tumors undergoing multidisciplinary skull base surgery.

Methods. A retrospective analysis was undertaken of children undergoing surgery at a single institution between 1998 and 2008 for benign and malignant lesions of the anterior, middle, or posterior cranial base. Patients with craniopharyngioma, pituitary tumors, and optic glioma were excluded. Histology, surgical morbidity, length of hospital stay, progression-free survival, and adjuvant therapy were recorded. Functional and cognitive outcome was assessed prospectively using the Late Effects Severity Score (LESS).

Results. Twenty-three children ranging in age from 13 months to 15 years underwent skull base approaches for resection of tumors during the study period. The median follow-up duration was 60 months. Tumor types included meningioma, schwannoma, rhabdomyosarcoma, neuroblastoma, angiofibroma, and chordoma. Complete resection was achieved in 12 patients (52\%). Thirteen patients (57\%) had benign histology. The median hospital stay was 7 days. There were 3 deaths, 1 perioperative and 2 from tumor progression. Two patients had CSF leakage (9\%) and 2 developed meningitis. Two children (9\%) had residual neurological deficit at last follow-up evaluation. Thirteen $(59 \%)$ of 22 surviving patients received adjuvant therapy. The majority of the patients remain in mainstream education and 19 of the 20 surviving children have an LESS of 3 or lower.

Conclusions. Children tolerate complex skull base procedures well, with minimal surgical-related morbidity as well as good long-term tumor control rates and functional outcomes from maximal safe resection combined with adjuvant treatment when required.
\end{abstract}

(http://thejns.org/doi/abs/10.3171/2013.1.PEDS12120)

KeY Words • skull base surgery • outcome • oncology

$\mathrm{O}$ UTCOMES for skull base surgery are well described in the adult population, but reports of surgery for lesions involving the skull base in children are rare. The pediatric skull base poses several unique problems to the surgeon, including the small size, location of unerupted dentition, a developing basicranium with varying location of key anatomical landmarks, and the potential for long-term sequelae to the developing craniofacial skeleton. In addition, due to the rarity of lesions requiring complex skull base approaches in children, there is little consensus on management, the need for radical resection, and ultimate outcome. There is increasing interest

Abbreviations used in this paper: $\mathrm{CPA}=$ cerebellopontine angle; GOS = Glasgow Outcome Scale; LESS = Late Effects Severity Score; NF2 = neurofibromatosis Type 2. in functional outcome in the adult skull base literature, in terms of the adverse impact of skull base approaches on quality of life, ${ }^{9}$ but to date there is a paucity of literature on functional outcome in children undergoing similar procedures.

The current study aims to review a single institution's experience of combined multidisciplinary skull base surgery in children younger than 16 years of age to assess outcome in terms of the impact of both approach-related and tumor-related morbidity on long-term functional outcome.

Study Population

\section{Methods}

Patients younger than 16 years of age who underwent 
multidisciplinary skull base procedures with a neurosurgeon and either an otolaryngologist or maxillofacial surgeon at Alder Hey Children's Hospital between 1998 and 2008 were identified from the surgical tumor registry. The study was approved by the institutional review board. Patients with craniopharyngioma, pituitary tumors, and optic glioma were excluded from the analysis.

\section{Data Collection}

All patient records were reviewed for presenting symptoms, surgical approach, and surgical complications. Extent of resection was defined by the operating surgeon and confirmed on immediate postoperative imaging. The total length of hospital stay was recorded as well as the use of adjuvant chemotherapy or radiotherapy. Episodes of tumor recurrence and treatment at recurrence were recorded. Progression-free survival and overall survival were documented.

\section{Outcome Assessment}

Functional outcome was assessed using academic attainment measures including highest level of education, mainstream schooling, or need for special educational services. Academic attainment was assessed at the last available follow-up evaluation for each patient. Additionally, the LESS (Table 1) was calculated for each patient, as the LESS has been shown to correlate with neurocognitive outcome. ${ }^{3}$

\section{Statistical Analyses}

All statistical analyses were performed with SPSS version 17 (SPSS Inc.). A probability $<0.05$ was defined as statistically significant.

\section{Results}

Twenty-three children ranging in age from 13 months to 15 years underwent multidisciplinary skull base approaches for resection of tumors during the study period (Table 2). The overall median age at presentation was 7 years: 4 years for malignant tumors and 9 years for benign tumors $(\mathrm{p}=0.31)$. The median follow-up duration was 60 months (range $6-156$ months). There were 13 boys and 10 girls. Thirteen patients (57\%) had benign histol- ogy. The majority of all skull base tumors $(61 \%)$ involved the anterior cranial fossa.

There were 3 deaths in the series. One patient died 3 days following presentation and surgery. During attempted resection of a posterior fossa/clival melanocytoma, uncontrollable hemorrhage occurred, the operation was abandoned after biopsy only, and the patient died 3 days later from intratumoral hemorrhage and swelling. Two other deaths occurred in the series, one at 18 months and one at 88 months, both from disseminated malignancy. No children in this series required any local or free-flap reconstruction. The median length of stay in the hospital was 7 days (range 2-46 days).

\section{Extent of Resection}

A gross-total resection was achieved in 12 cases (52\%). A gross-total resection was achieved in 12 (80\%) of 15 patients in which gross-total resection was the predefined surgical goal (Figs. 1 and 2). In the 3 cases in which a complete resection was not achieved, all involved tumor in the cavernous sinus and clival carotid region (2 angiofibromas and 1 chordoma), where a small remnant was left adherent to the carotid.

In the 8 cases in which the surgical plan was subtotal resection, 1 patient had an extensive parasellar meningioma with NF2. There was bilateral cavernous sinus involvement with this tumor and the goal was maximal safe resection with functional preservation. A child with a suprasellar reparative granuloma underwent debulking, but there was diffuse bone involvement that was managed with oral calcitonin. Similarly, the child with fibrous dysplasia had widespread bone involvement and underwent resection of the sphenoorbital component and was subsequently treated with bisphosphonates. The remaining 5 children had suspected high-grade tumors and were managed according to contemporaneous protocols of the United Kingdom Children's Cancer and Leukaemia Group, involving subtotal resection and adjuvant treatment. In such cases the surgical aims will be determined at the relevant multidisciplinary team meeting, which also applies to other nonmalignant tumor cases, and will be based on the tumor protocol, individual risk factors, and with consideration of the administration of chemotherapy and/or radiotherapy. In this respect the aim of surgery is

TABLE 1: Late Effects Severity Score (LESS) scale*

\begin{tabular}{|c|c|c|}
\hline Category & 1 Point & 2 Points \\
\hline neurology & $\begin{array}{l}1 \text { isolated neurological late effect (mild ataxia, mild hemi- } \\
\text { paresis, cranial nerve palsy); pathological electroen- } \\
\text { cephalography }\end{array}$ & $\begin{array}{l}\text { any combination of }>1 \text { neurological late effect; sei- } \\
\text { zures }\end{array}$ \\
\hline endocrine & unsubstituted hormone deficit & $\geq 1$ hormone deficit \\
\hline visual/auditory & $\begin{array}{l}\text { uni- or bilat hearing impairment not requiring aid \&/or } \\
\text { visual impairment }\end{array}$ & $\begin{array}{l}\text { unilat deafness or any hearing impairment requiring } \\
\text { aid \&/or visual impairment requiring special train- } \\
\text { ing/equipment }\end{array}$ \\
\hline others & $\begin{array}{l}\text { any treatment-related medical problem not listed, but not } \\
\text { requiring medical intervention }\end{array}$ & $\begin{array}{l}\text { any treatment-related medical problem not listed, but } \\
\text { requiring medical intervention (such as a VP } \\
\text { shunt) }\end{array}$ \\
\hline
\end{tabular}

* VP = ventriculoperitoneal. 
C. Hayhurst et al.

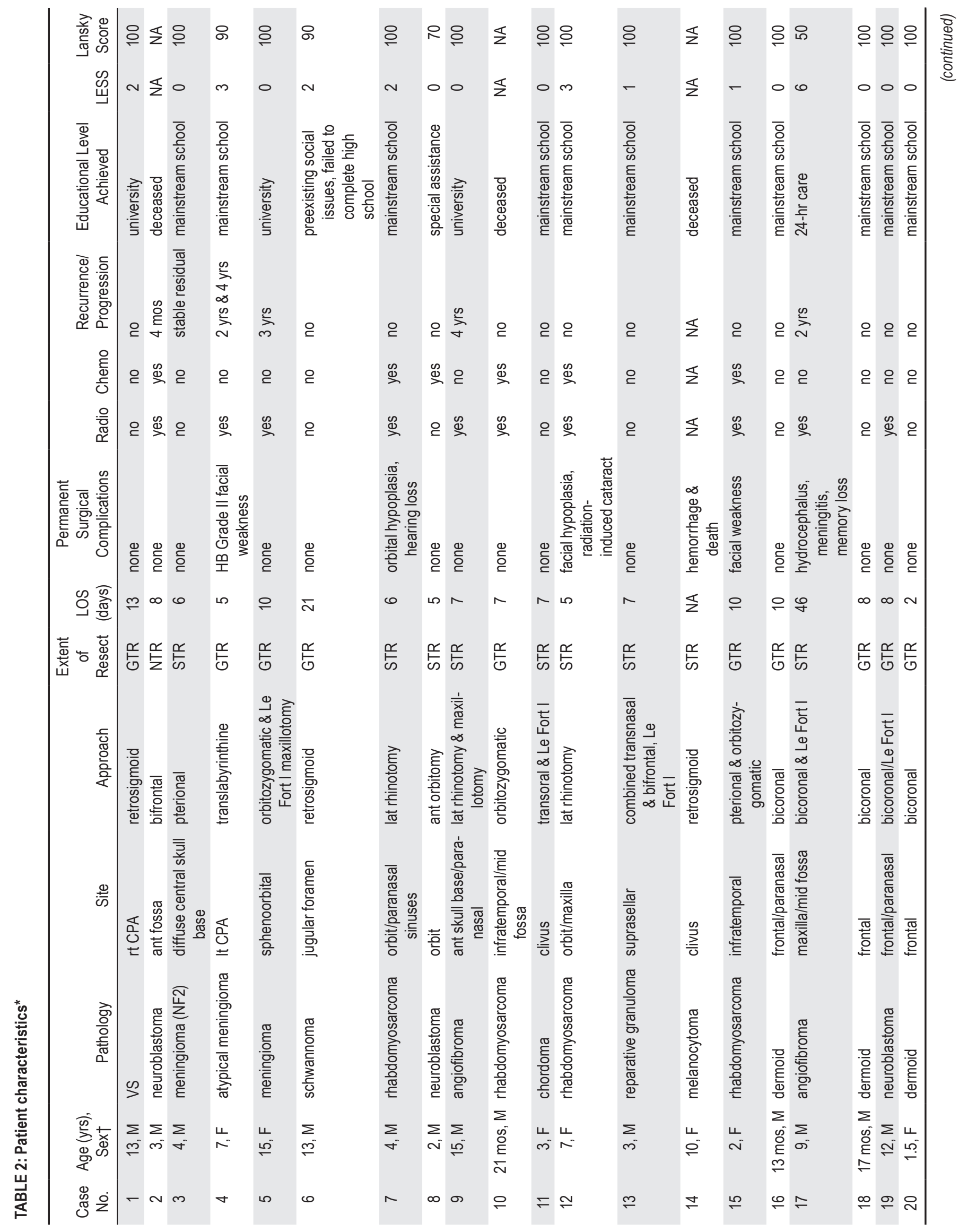




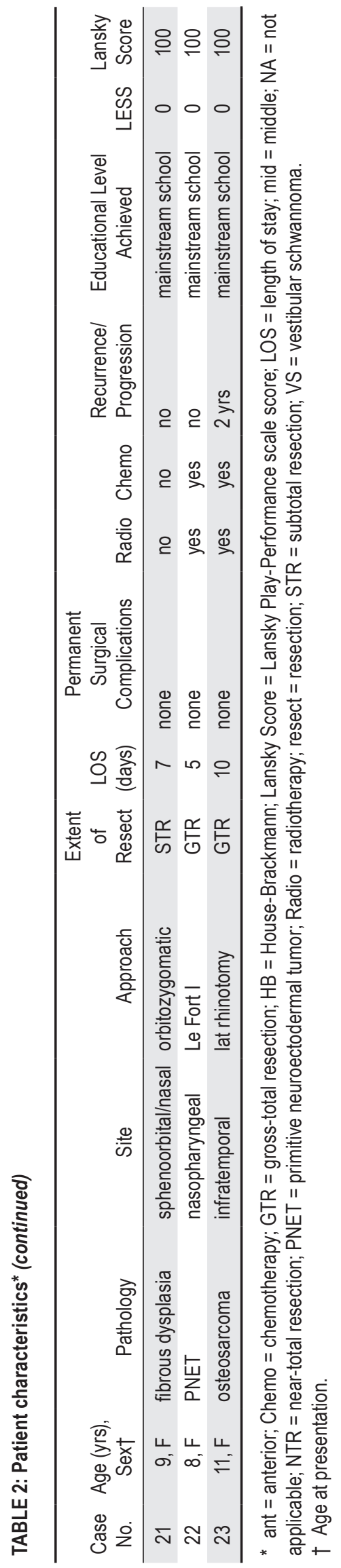

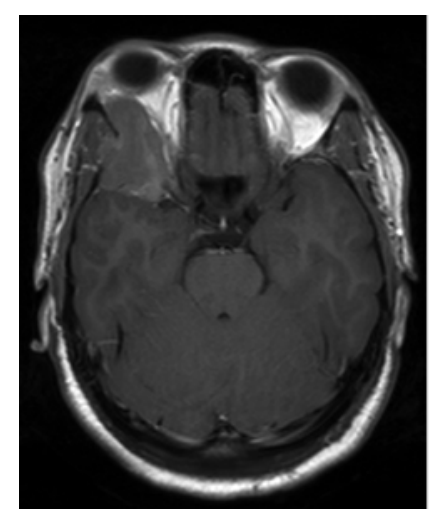

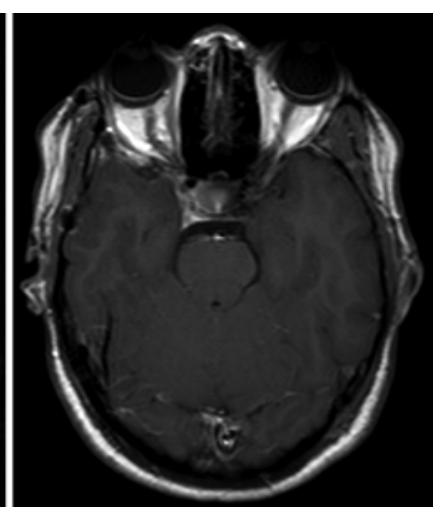

FIG. 1. Case 5. Axial T1-weighted MR images with Gd enhancement demonstrating a right sphenoorbital meningioma (left) and complete tumor excision from the right sphenoid with residual high signal intensity from fat grafting (right).

often to debulk the tumor and thus not always to obtain a complete tumor resection.

\section{Technological Adjuncts to Surgery}

This case series represents several years of evolving refinements to surgery. All cases were performed using neuronavigation, either optical or, since 2009, electromagnetic. All lateral and posterior skull base procedures were performed with neuromonitoring. Recently we have introduced intraoperative MRI, which has proven useful in skull base procedures. Case 5 was one of the first cases operated on in the intraoperative MRI suite at our institution. This patient underwent an orbitozygomatic approach for a sphenoorbital meningioma. Once resection was believed to be complete she underwent intraoperative imaging, which revealed residual tumor in the lateral recess of the sphenoid sinus that had been missed and was subsequently completely resected (Fig. 3).

\section{Surgical Complications}

Surgery-related complications (transient and permanent) occurred in 7 patients (30.4\%), including CSF leak in 2 patients $(9 \%)$, meningitis in 2 patients $(9 \%)$, and transient nerve palsy in the sixth cranial nerve in 1 patient.
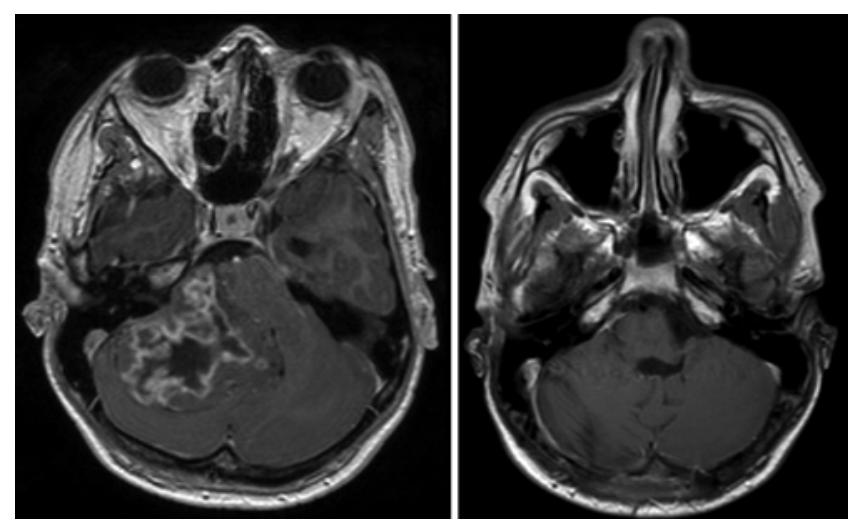

FIG. 2. Case 6. Axial T1-weighted MR images with Gd enhancement demonstrating a right CPA tumor with significant cerebellar and brainstem compression (left) and complete excision of the jugular schwannoma postoperatively (right). 


\section{Hayhurst et al.}

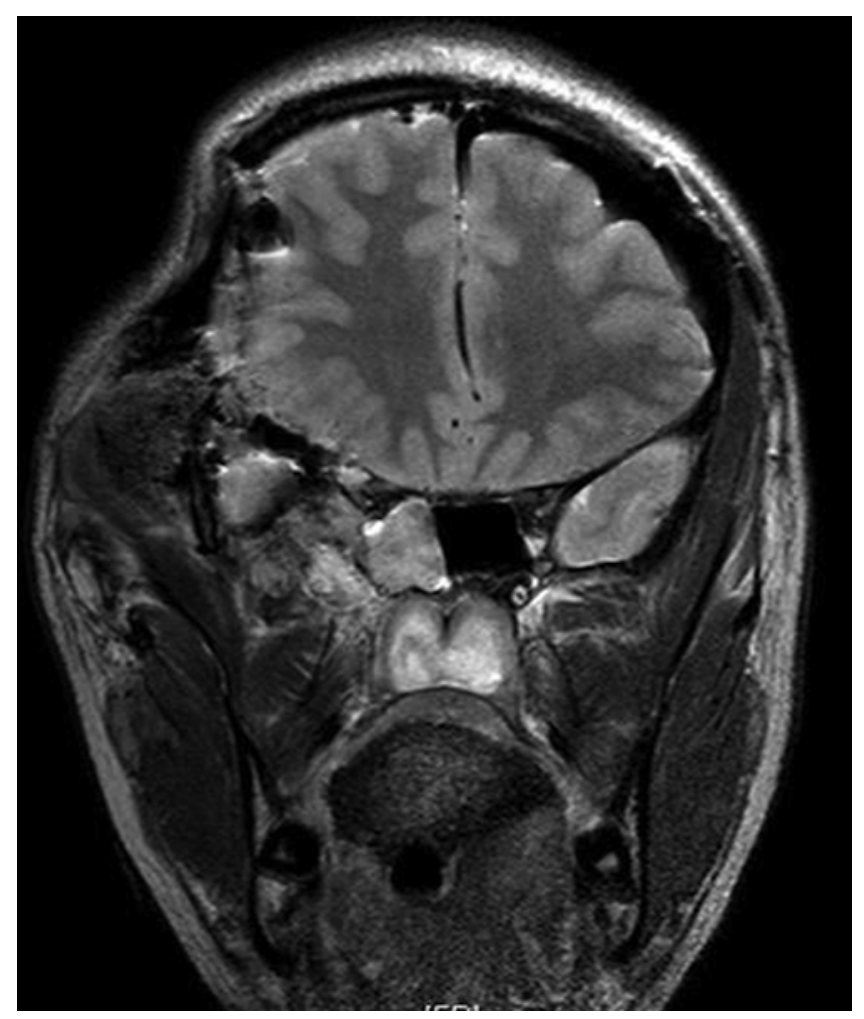

FIG. 3. Case 5. Intraoperative coronal 3-T MR image showing residual meningioma in the sphenoid sinus.

One patient suffered a cerebellar infarct but without permanent neurological sequelae.

Three (13\%) of 23 children required a ventriculoperitoneal shunt following surgery. However, only 2 patients experienced unanticipated permanent neurological deficits following surgery. One patient has a House-Brackmann Grade II facial weakness following resection of a CPA meningioma and 1 patient developed memory loss. Three children had expected postoperative deficits, 2 with hearing loss following resection of CPA lesions and 1 child underwent sacrifice of the facial nerve during tumor resection (Case 15).

\section{Adjuvant Treatment}

Overall 6 (27\%) of the 22 children who survived surgery had recurrence or progression of their tumor following initial treatment. Of those 6 children, 2 had malignant disease and 4 had histologically benign disease (angiofibroma and meningioma). Three patients underwent 1 further surgery and 1 patient had 2 further operations. All patients were treated with radiotherapy at the time of recurrence and 1 patient with rhabdomyosarcoma underwent chemotherapy at the time of tumor progression.

In total $13(59 \%)$ of 22 children underwent adjuvant therapy. All patients with high-grade tumors $(n=9)$ were given adjuvant treatment according to Children's Cancer and Leukaemia Group guidelines (Fig. 4), with chemotherapy only given to a child with orbital neuroblastoma presenting at age 2 years. In the 4 other cases radiotherapy was reserved for tumor progression, where further surgery was undertaken, and they were then treated with radiotherapy after repeat surgery.

In 2 cases a subtotal resection was performed but no adjuvant treatment was given. In 1 case of an extensive parasellar meningioma in a 4-year-old child with NF2, it is our policy to avoid radiotherapy in the setting of NF2, and the residual tumor has been stable on serial imaging for 9 years. A 3-year-old girl with a small residual clival chordoma (Case 11) was offered referral for proton beam radiation therapy but the parents declined and the residual tumor has been stable on serial imaging for 7 years.

\section{Progression-Free and Overall Survival}

Overall survival was $87 \%$ (20 of 23) at a median of 60 months. The overall 1- and 5-year survival rates in children with benign disease were both $92 \%$, whereas the 1- and 5-year survival rates for children with malignant disease were $100 \%$ and $90 \%$, respectively. Progressionfree survival was $95 \%$ at 1 year $(90 \%$ malignant disease and $100 \%$ benign disease) and $68 \%$ at 5 years (70\% malignant disease and $66 \%$ benign disease).

\section{Late Effects and Functional Outcome}

The LESS for each surviving child is listed in Table 2 and compared with the Lansky Play-Performance scale score. Ninety-five percent of the children scored 3 or lower on the LESS, indicating minimal late effects from surgery or adjuvant treatment. In contrast, a 9-year-old boy (Case 17) who underwent surgery for an extensive maxillary and middle fossa angiofibroma suffered severe postoperative complications including meningitis, hydrocephalus, and significant memory impairment, and had panhypopituitarism following treatment. This boy had an LESS of 6 and requires 24-hour home care.

Three (15\%) of the 20 surviving children have permanent pituitary dysfunction. In 2 children this dysfunction was as a result of adjuvant treatment, and in 1 child who has partial anterior hypopituitarism following resection of a suprasellar granuloma. Of the 20 surviving children, $17(85 \%)$ remain in mainstream schooling without special educational assistance. One child who underwent surgery and chemotherapy for neuroblastoma at age 2 requires special assistance at a mainstream school. One child with a jugular foramen schwannoma who underwent complete excision complicated by meningitis and hydrocephalus requiring a ventriculoperitoneal shunt failed to complete high school after recovery, largely due to preexisting social issues. Three of the patients in the series have now reached the age of 18 and all are enrolled in university education, and all had benign disease. Two children with paraorbital rhabdomyosarcoma have a mild degree of ipsilateral facial hypoplasia, but no other facial skeletal growth abnormalities have been observed in this series.

\section{Discussion}

In this series, children tolerated extensive combined craniofacial and skull base approaches well, with minimal functional impairment and late effects, despite the high use of adjuvant therapy (59\%). Although a gross-total resection was only achieved in 52\%, the overall survival 

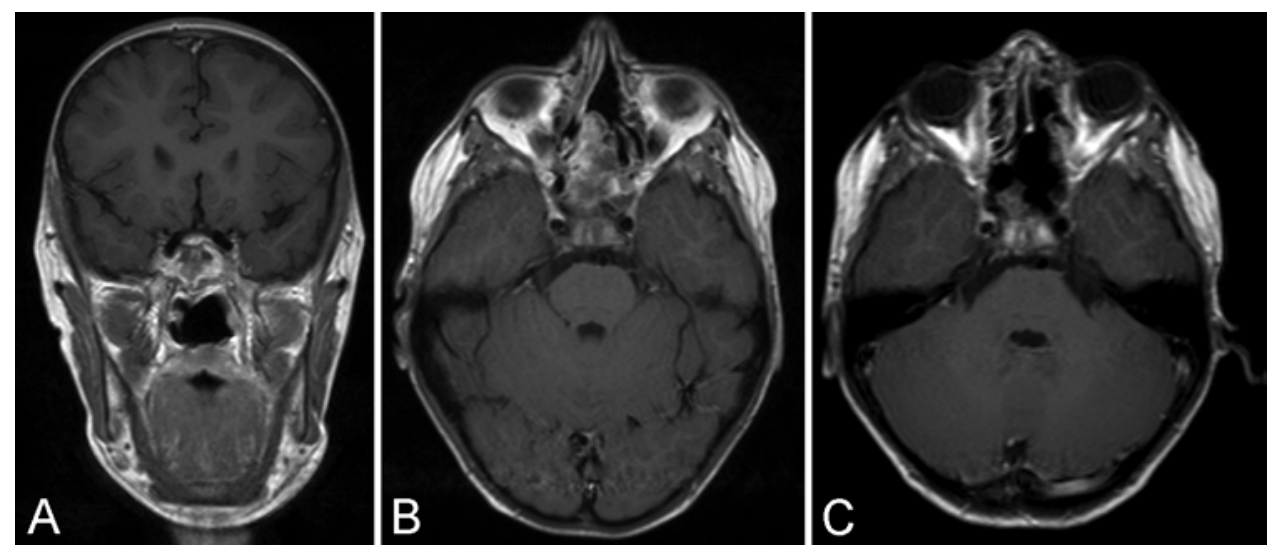

FIG. 4. Case 19. Preoperative and postoperative T1-weighted MR images of an olfactory neuroblastoma. A: Coronal image demonstrating a soft-tissue mass (in the sphenoid sinus and posterior nasopharyngeal space). B: Axial image demonstrating a soft-tissue mass in the posterior nasopharynx extending into the sphenoid sinus. C: Axial image with Gd enhancement showing complete tumor excision from the posterior nasopharynx with evidence of residual mucosal thickening in the postnasal space and sphenoid sinus. Postoperative radiotherapy was administered.

rate at a median follow-up of 60 months was $87 \%$, and the rate of permanent neurological morbidity was $9 \%$.

There are few studies in the literature on long-term outcome in children undergoing complex skull base surgery. ${ }^{6,8,11,14,17,18,22,26}$ The largest series reported by Brockmeyer et al. ${ }^{6}$ included 55 children who underwent skull base approaches for a variety of lesions, including 41 tumors and 2 fibrous dysplasias, in addition to vascular lesions and epidural abscess. The majority of patients did well, with an $11 \%$ rate of permanent neurological morbidity. Brockmeyer et al. ${ }^{6}$ used the GOS to assess outcome objectively and $96 \%$ had a GOS score of 4 or 5 . Lang et al. ${ }^{18}$ reported on 20 children with a range of pathologies including juvenile nasopharyngeal angiofibroma, chordoma, schwannoma, and meningioma who underwent craniofacial access procedures. They also used GOS to assess outcome, with $90 \%$ having a good outcome. One patient was moderately disabled and 1 child severely disabled. In addition, Lang et al. ${ }^{18}$ reported educational status at last follow-up: 18 of 20 patients resumed mainstream education and 2 had existing preoperative difficulties requiring educational assistance.

Teo et al. ${ }^{26}$ reviewed 26 children who underwent skull base approaches for various tumors, including schwannoma, fibrous dysplasia, chordoma, and esthesioneuroblastoma. The rate of immediate postoperative complications was $57 \%$ and permanent complications occurred in $30.7 \%$. These investigators reported a $92 \%$ complete resection rate and an $81 \%$ tumor-free survival rate at 2 years.

The majority of published case series of pediatric skull base approaches reported a relatively high rate of complications in the immediate postoperative period but a much lower permanent neurological complication rate ranging from $0 \%$ to $14 \%,{ }^{6,8,10,11,14,22}$ reflecting the plasticity of the developing nervous system. In general it is believed that outcomes in pediatric skull base surgery are better than in adult patients, attributed to higher rates of complete resection afforded by better tissue planes, more frequent benign pathology, and centralization in high-volume pediatric centers. ${ }^{26,27}$ However, the need for radical resection at the cost of additional morbidity is uncertain.
Hanbali et al. ${ }^{14}$ reviewed 24 children with tumors arising from the skull base who required surgical management, including 11 benign tumors and 13 malignant tumors. Six (25\%) of 24 patients underwent subtotal resection or biopsy at the first surgery. Thirteen patients received chemotherapy, radiation therapy, or both, either prior to surgery at their institution or at the time of progression. Forty-two percent of children experienced postoperative complications, but persistent neurological morbidity occurred in $8 \%$. There was 1 death at 30 days from septicemia. The overall 1- and 5-year survival rates were both $87 \%$. Hanbali et al..$^{14}$ advocate radical resection without adjuvant therapy for optimal treatment of benign and low-grade malignant lesions such as chordoma.

Kalani et al. ${ }^{15}$ reported on 22 patients with a mean age of 15 years who underwent anterior craniofacial disassembly for large juvenile nasopharyngeal angiofibromas, with a complete resection achieved in $77 \%$. The authors state that their philosophy is to resect accessible tumor but to leave behind intracavernous tumor, as in our cases. In their series, Kalani et al. ${ }^{15}$ reported a $10 \%$ incidence of musculoskeletal defects requiring delayed surgical correction and the overall rate of postoperative complications was $41 \%$, mainly CSF leaks and infection.

In our series, 10 children underwent transfacial procedures, including 6 Le Fort I approaches. Two children have mild orbital hypoplasia, and both of them underwent a lateral rhinotomy for rhabdomyosarcoma at ages 7 and 4, respectively. Both patients received adjuvant radiotherapy. Orbital hypoplasia has been reported in $56 \%$ of children treated with radiotherapy for rhabdomyosarcoma of the orbit. $^{12}$ No other facial growth abnormalities were noted in this series. In their series of 20 children following craniofacial access procedures, Lang et al. ${ }^{18}$ did not detect any disruption to facial growth at a mean follow-up of 2.8 years. Lewark et al. ${ }^{19}$ reported the loss of tooth buds in children younger than 5 who underwent a Le Fort I osteotomy, but they did not observe problems with facial growth. Demonte et al. ${ }^{7}$ described 12 children who underwent resection of skull base tumors and required reconstruction. In their series, 1 patient who was 18 months of age had asymmet- 


\section{Hayhurst et al.}

rical facial growth following a zygomatic osteotomy, and Demonte et al. advocate the avoidance of zygomatic osteotomies in children younger than 4 years. However, Miller et al. ${ }^{23}$ reported 6 cases treated using a modified orbitozygomatic craniotomy with an age range of 26 months to 15 years and found no impact on postoperative cosmesis with preservation of temporalis muscle bulk. A study on facial development following excision of anterior skull base tumors in children used cephalometric analysis to estimate facial growth. ${ }^{21}$ Overall, this study showed that cephalometric changes were with $10 \%$ of normal values with no adverse effect on cosmetic outcome.

Refinements in technological advances applied to adult skull base surgery also increase the safety and efficacy of pediatric skull base surgery, including image guidance, ${ }^{2,4}$ intraoperative $\mathrm{MRI}^{1}$ (as described in the current study), and endoscopy. ${ }^{16,20,25}$ Because a large proportion of pediatric skull base pathology arises in the anterior cranial fossa, ${ }^{8,14}$ endoscopic endonasal approaches are likely to play an increasing role. ${ }^{24}$ Pathologies specific to the pediatric population such as juvenile nasopharyngeal angiofibroma are increasingly managed entirely endoscopically, with case series demonstrating excellent local tumor control. ${ }^{5,13}$ Kassam et al. ${ }^{16}$ reported 25 pediatric cases from 430 consecutive endoscopic endonasal procedures, demonstrating the safety and feasibility of the endoscopic endonasal approach for midline skull base pathology such as juvenile nasopharyngeal angiofibroma, pituitary masses, and chordoma in children as young as 3 years of age.

Of the surviving children in our series, $85 \%$ remain in mainstream schooling and $95 \%$ had an LESS of 3 or lower. The LESS was developed to numerically quantify treatment-related sequelae in children who underwent chemoradiotherapy for medulloblastoma or ependymoma. ${ }^{3}$ It has a maximum score of 8 across 4 domains of neurology, endocrinology, visual/auditory, and others. The LESS has been shown to correlate with neurocognitive outcome in children with malignant brain tumors, but has less correlation with self-reported measures of quality of life. ${ }^{3}$ This is the first report of the use of LESS in a mixed group of malignant and benign skull base lesions, although in this series a high proportion underwent adjuvant chemoradiotherapy. The utility of LESS in this context should be explored prospectively in a larger series of patients undergoing skull base surgery. Although LESS can provide a surrogate measure of functional outcome, future studies should also include prospective measures of quality of life such as the Pediatric Quality of Life Inventory (PedsQL) and European Organisation for Research and Treatment of Cancer Quality of Life Questionnaire (EORTC QLQ-C30) in children undergoing extensive cranial resections.

\section{Conclusions}

Using a combined multidisciplinary approach, children tolerate skull base surgery well with a low rate of permanent neurological morbidity and minimal late effects. Future prospective studies including neuropsychological and quality of life measures are needed to assess the full impact of skull base tumors, both malignant and benign, on child development.

\section{Disclosure}

The authors report no conflict of interest concerning the materials or methods used in this study or the findings specified in this paper.

Author contributions to the study and manuscript preparation include the following. Conception and design: Hayhurst. Acquisition of data: Williams, Yousaf. Analysis and interpretation of data: Hayhurst. Drafting the article: Hayhurst, Richardson. Critically revising the article: all authors. Reviewed submitted version of manuscript: all authors. Approved the final version of the manuscript on behalf of all authors: Hayhurst. Statistical analysis: Williams. Administrative/technical/material support: Yousaf. Study supervision: Pizer, Mallucci.

\section{References}

1. Abernethy LJ, Avula S, Hughes GM, Wright EJ, Mallucci CL: Intra-operative 3-T MRI for paediatric brain tumours: challenges and perspectives. Pediatr Radiol 42:147-157, 2012

2. Alshail E, Rutka JT, Drake JM, Hoffman HJ, Humphreys R, Phillips J, et al: Utility of frameless stereotaxy in the resection of skull base and Basal cerebral lesions in children. Skull Base Surg 8:29-38, 1998

3. Benesch M, Spiegl K, Winter A, Passini A, Lackner H, Moser A, et al: A scoring system to quantify late effects in children after treatment for medulloblastoma/ependymoma and its correlation with quality of life and neurocognitive functioning. Childs Nerv Syst 25:173-181, 2009

4. Benoit MM, Silvera VM, Nichollas R, Jones D, McGill T, Rahbar R: Image guidance systems for minimally invasive sinus and skull base surgery in children. Int J Pediatr Otorhinolaryngol 73:1452-1457, 2009

5. Bleier BS, Kennedy DW, Palmer JN, Chiu AG, Bloom JD, O'Malley BW Jr: Current management of juvenile nasopharyngeal angiofibroma: a tertiary center experience 1999-2007. Am J Rhinol Allergy 23:328-330, 2009

6. Brockmeyer D, Gruber DP, Haller J, Shelton C, Walker ML: Pediatric skull base surgery. 2. Experience and outcomes in 55 patients. Pediatr Neurosurg 38:9-15, 2003

7. Demonte F, Moore BA, Chang DW: Skull base reconstruction in the pediatric patient. Skull Base 17:39-51, 2007

8. Gil Z, Constantini S, Spektor S, Abergel A, Khafif A, BeniAdani L, et al: Skull base approaches in the pediatric population. Head Neck 27:682-689, 2005

9. Gil Z, Fliss DM: Quality of life in patients with skull base tumors: current status and future challenges. Skull Base 20: $11-18,2010$

10. Gil Z, Patel SG, Cantu G, Fliss DM, Kowalski LP, Singh B, et al: Outcome of craniofacial surgery in children and adolescents with malignant tumors involving the skull base: an international collaborative study. Head Neck 31:308-317, 2009

11. Gross ND, Ganly I, Patel SG, Bilsky MH, Shah JP, Kraus DH: Results of anterior skull base surgery in pediatric and young adult patients. Skull Base 20:75-81, 2010

12. Guyuron B, Dagys AP, Munro IR, Ross RB: Effect of irradiation on facial growth: a 7- to 25-year follow-up. Ann Plast Surg 11:423-427, 1983

13. Hackman T, Snyderman CH, Carrau R, Vescan A, Kassam A: Juvenile nasopharyngeal angiofibroma: the expanded endonasal approach. Am J Rhinol Allergy 23:95-99, 2009

14. Hanbali F, Tabrizi P, Lang FF, DeMonte F: Tumors of the skull base in children and adolescents. J Neurosurg 100 (2 Suppl Pediatrics):169-178, 2004

15. Kalani MY, Kalani MA, Kalb S, Albuquerque FC, McDougall CG, Nakaji P, et al: Craniofacial approaches to large juve- 


\section{Pediatric skull base surgery}

nile angiofibromas. Clinical article. J Neurosurg Pediatr 8: 71-78, 2011

16. Kassam A, Thomas AJ, Snyderman C, Carrau R, Gardner P, Mintz A, et al: Fully endoscopic expanded endonasal approach treating skull base lesions in pediatric patients. J Neurosurg 106 (2 Suppl):75-86, 2007

17. Kennedy JD, Haines SJ: Review of skull base surgery approaches: with special reference to pediatric patients. J Neurooncol 20:291-312, 1994

18. Lang DA, Neil-Dwyer G, Evans BT, Honeybul S: Craniofacial access in children. Acta Neurochir (Wien) 140:33-40, 1998

19. Lewark TM, Allen GC, Chowdhury K, Chan KH: Le Fort I osteotomy and skull base tumors: a pediatric experience. Arch Otolaryngol Head Neck Surg 126:1004-1008, 2000

20. Locatelli D, Castelnuovo P, Santi L, Cerniglia M, Maghnie M, Infuso L: Endoscopic approaches to the cranial base: perspectives and realities. Childs Nerv Syst 16:686-691, 2000

21. Lowlicht RA, Jassin B, Kim M, Sasaki CT: Long-term effects of Le Fort I osteotomy for resection of juvenile nasopharyngeal angiofibroma on maxillary growth and dental sensation. Arch Otolaryngol Head Neck Surg 128:923-927, 2002

22. Mandonnet E, Kolb F, Tran Ba Huy P, George B: Spectrum of skull base tumors in children and adolescents: a series of 42 patients and review of the literature. Childs Nerv Syst 24: 699-706, 2008
23. Miller ML, Kaufman BA, Lew SM: Modified osteoplastic orbitozygomatic craniotomy in the pediatric population. Childs Nerv Syst 24:845-850, 2008

24. Munson PD, Moore EJ: Pediatric endoscopic skull base surgery. Curr Opin Otolaryngol Head Neck Surg 18:571-576, 2010

25. Pirris SM, Pollack IF, Snyderman CH, Carrau RL, Spiro RM, Tyler-Kabara E, et al: Corridor surgery: the current paradigm for skull base surgery. Childs Nerv Syst 23:377-384, 2007

26. Teo C, Dornhoffer J, Hanna E, Bower C: Application of skull base techniques to pediatric neurosurgery. Childs Nerv Syst 15:103-109, 1999

27. Tsai EC, Santoreneos S, Rutka JT: Tumors of the skull base in children: review of tumor types and management strategies. Neurosurg Focus 12(5):e1, 2002

Manuscript submitted February 29, 2012.

Accepted January 17, 2013.

Please include this information when citing this paper: published online February 22, 2013; DOI: 10.3171/2013.1.PEDS12120.

Address correspondence to: Caroline Hayhurst, F.R.C.S., Department of Neurosurgery, University Hospital of Wales, Heath Park, Cardiff CF14 4XW, United Kingdom. email: carolinehayhurst@ hotmail.com. 